\title{
Expression Analysis of Hairpin RNA Carrying Sugarcane mosaic virus (SCMV) Derived Sequences and Transgenic Resistance Development in a Model Rice Plant
}

\author{
Sehrish Akbar, ${ }^{1}$ Muhammad Tahir, ${ }^{1}$ Ming-Bo Wang, ${ }^{2}$ and Qing Liu ${ }^{2}$ \\ ${ }^{1}$ Atta-ur-Rahman School of Applied Biosciences (ASAB), National University of Sciences and Technology (NUST), \\ Islamabad 44000, Pakistan \\ ${ }^{2}$ Commonwealth Scientific Industrial and Research Organization, Agriculture, GPO Box 1600, Canberra, ACT 2601, Australia
}

Correspondence should be addressed to Muhammad Tahir; gullsbs@outlook.com

Received 2 August 2016; Revised 8 December 2016; Accepted 9 January 2017; Published 1 February 2017

Academic Editor: Rita Casadio

Copyright (C) 2017 Sehrish Akbar et al. This is an open access article distributed under the Creative Commons Attribution License, which permits unrestricted use, distribution, and reproduction in any medium, provided the original work is properly cited.

\begin{abstract}
Developing transgenic resistance in monocotyledonous crops against pathogens remains a challenging area of research. Sugarcane mosaic virus (SCMV) is a serious pathogen of many monocotyledonous crops including sugarcane. The objective of present study was to analyze transgenic expression of hairpin RNA (hpRNA), targeting simultaneously CP (Coat Protein) and Hc-Pro (helper component-proteinase) genes of SCMV, in a model rice plant. Conserved nucleotide sequences, exclusive for DAG (Aspartic acid-Alanine-Glycine) and KITC (Lycine-Isoleucine-Threonine-Cysteine) motifs, derived from SCMV CP and Hc-Pro genes, respectively, were fused together and assembled into the hpRNA cassette under maize ubiquitin promoter to form Ubi-hpCP:Hc-Pro construct. The same CP:Hc-Pro sequence was fused with the $\beta$-glucuronidase gene (GUS) at the $3^{\prime}$ end under CaMV 35 S promoter to develop 35S-GUS:CP:Hc-Pro served as a target reporter gene construct. When delivered into rice callus tissues by particle bombardment, the Ubi-hpCP:Hc-Pro construct induced strong silencing of 35S-GUS:CP:Hc-Pro. Transgenic rice plants, containing Ubi-hpCP:Hc-Pro construct, expressed high level of 21-24 nt small interfering RNAs, which induced specific suppression against GUS:CP:Hc-Pro delivered by particle bombardment and conferred strong resistance to mechanically inoculated SCMV. It is concluded that fusion hpRNA approach is an affordable method for developing resistance against SCMV in model rice plant and it could confer SCMV resistance when transformed into sugarcane.
\end{abstract}

\section{Introduction}

Sugarcane mosaic virus (SCMV), belonging to genus Potyvirus, family Potyviridae, is a serious pathogen of many monocotyledonous crops including sugarcane. In Pakistan, approximately $10-32 \%$ losses has been estimated in sugarcane yield which results in 6-10\% decline in sugar production [1]. Infected plants showed mosaic pattern and are characterized by typical pinwheel shaped inclusion bodies in cell cytoplasm. The $10 \mathrm{~kb}$ single stranded RNA genome of SCMV encodes a single polypeptide which is cleaved either co- or post translationally into ten mature proteins ( $P 1, H C-P r o, P 3,6 K 1$, $\mathrm{CI}, 6 \mathrm{~K} 2, \mathrm{VPg}, \mathrm{NIa}-\mathrm{Pro}, \mathrm{NI}$, and $\mathrm{CP})[2,3]$. C-terminus of the polyprotein encodes the coat protein $(\mathrm{CP})$ which encapsidates the viral genome through helical arrangement of its multiple subunits [4]. Hc-Pro is a multifunctional protein and a proteinase, which is responsible for the multiplication of virus genome, systemic virus movement [5], and suppression of plant RNA silencing machinery [6] by interacting with numerous host factors.

A conserved DAG (Aspartic acid-Alanine-Glycine) motif of $C P$ is responsible for aphid mediated transmission of virus in combination with $\mathrm{Hc}$-Pro which has a conserved KITC (Lycine-Isoleucine-Threonine-Cysteine) motif that binds in aphid stylet [7]. There are two hypotheses about aphid mediated Potyvirus transmission, including the bridge hypothesis and the direct hypothesis. The bridge hypothesis reported that the N-terminal region of Hc-Pro (KITC motif) recognizes an unknown receptor in the aphid stylets. At the same time or subsequently, an Hc-Pro downstream motif, 
presumably containing the PTK motif, undergoes a specific interaction with the DAG motif on the virus $C P$, thereby mediating retention of the virions at appropriate sites in the vector [8].

Over the last few decades, considerable efforts have been made to control SCMV using diverse approaches. Pathogen derived resistance through coat protein mediated resistance has been used extensively to develop resistance against different virus groups. It has been reported that sense SCMV CP gene construct transformed into sugarcane showed different level of resistance when challenged with SCMV [9]. Furthermore, multiple sugarcane lines were also generated through the transformation of the coding sequence of CP gene of Sorghum mosaic virus (SrMV) strain $\mathrm{H}$ into sugarcane. However, some of these sugarcane transgenic lines displayed the mosaic symptoms after being challenged with SCMV-H in field trials [10].

RNA silencing is an evolutionarily conserved gene regulation mechanism in eukaryotes, which plays a fundamental role in controlling both regulation of endogenous gene expression and defense against invasive nucleic acids such as viruses and transposable elements [11, 12]. RNA silencing is induced by double stranded RNA (dsRNA) or hairpin RNA (hpRNA), which is processed into 21-24 nucleotide (nt) small interfering RNA (siRNA) duplex by Dicer or Dicer-like (DCL) protein. One strand of the siRNA duplex is incorporated into the Argonaute protein to form RNAInduced Silencing Complex (RISC) and guides RISC to single-stranded RNA via sequence complementarity, resulting in Argonaute-mediated cleavage of the target RNA [11, 13].

hpRNA-induced silencing has been established to be a powerful tool to developing plant viral resistance through the silencing of viral RNA. hpRNA targeting SrMV CP gene showed approximately $87.5 \%$ resistance against SrMV in transgenic sugarcane plants [14]. Evidence about long hpRNA construct targeting multiple genes of Rice black streaked dwarf virus was successfully used to regenerate stable and resistant lines against virus in rice [15].

This paper reports the expression analysis of hpRNA, targeting simultaneously $C P$ and Hc-Pro genes of SCMV, in a model rice plant. The approach was designed to generate SCMV resistance by silencing viral genes that play roles in virus transmission, encapsulation and multiplication, and counter defence against RNA silencing. The validation of the approach not only will lead to the development of SCMV resistant sugarcane but also will provide valuable material to developing resistance in other crops.

\section{Materials and Methods}

2.1. Preparation of the Ubi-hpCP:Hc-Pro Construct. Consensus sequences of CP and Hc-Pro genes of SCMV, $240 \mathrm{bp}$ each, were selected and fused into a chimeric fragment for use as the trigger DNA sequence in the hpRNA cassette. These targeted regions were selected by retrieving the multiple sequences of $C P$ and Hc-Pro genes (about 50 sequences for each gene) from NCBI database and aligned by using ClustalW [16]. A consensus sequence (240 bp) from each gene was selected to form the fusion sequence. This CP:Hc-Pro fusion sequence was synthesized by GeneArt $^{\mathrm{TM}}$ Gene Synthesis (Thermo Fisher Scientific, Waltham, MA) (Figure S1 in Supplementary Material available online at https://doi.org/10.1155/2017/1646140) and assembled into the hpRNA cassette in both sense and antisense gene orientations. For directional cloning into the pStarling vector, restriction sites of KpnI/SpeI and BamHI/SmaI were incorporated at $5^{\prime}$ and $3^{\prime}$ ends of fusion fragment, respectively. The expression cassette in pStarling was excised with NotI and inserted into pWBVec8 binary plant expression vector [17] (Figure 1(a)). The vector was transformed into Agrobacterium tumefaciens AGL1 strain by electroporation method for plant transformation. pWBVec8 binary vector [17] was also transformed as an empty vector control.

\subsection{Preparation of 35S-GUS: CP-Hc-Pro Fusion Target Con-} struct. The synthesized CP: Hc-Pro fusion fragment was transcriptionally fused with coding sequence of Escherichia coli $\beta$-glucuronidase gene (GUS) which was subcloned into pART7 [18] at the BamHI and XhoI sites between the CaMV $35 \mathrm{~S}$ promoter and the octopine synthase (OCS) terminator. The cassette was then excised with NotI and cloned into pWBVec2a to form the binary vector suitable for Agrobacteriummediated transformation (Figure 1(b)).

2.3. Rice Transformation. The seed of Nipponbare variety of rice (Oryza sativa) was selected and cultured on N6D medium for callus induction. Calli were transformed by the Ubi-hpCP:Hc-Pro and empty vector constructs using Agrobacterium-mediated transformation according to Hiei et al. [19].

\subsection{Particle Bombardment of Rice Calli and Transgenic Rice} Leaves. Nipponbare rice seed were germinated on N6D (Rice callus induction) medium [19] for 4-6 weeks. Healthy looking rice calli or leaves of transgenic rice were selected for particle bombardment. Plasmid DNA $(1 \mu \mathrm{g})$ was mixed with gold particles in $0.6 \mu$ in diameter, and $2.5 \mathrm{M} \mathrm{CaCl}_{2}(16 \mu \mathrm{L})$ was added, which aids the binding of negatively charged DNA molecules to gold particles. After brief vortexing, $0.1 \mathrm{M}$ spermidine solution $(6.4 \mu \mathrm{L})$ was added into the mixture for protection from endonucleases. These DNA-coated gold particles were bombarded into rice calli or leaves using PDS $1000-H e$ Biolistic particle delivery system at 1100 Psi pressure and 26 inch $\mathrm{Hg}$ vacuum at $6 \mathrm{~cm}$ optimized target distance. GUS expression was analyzed using histochemical staining 2-3 days after bombardment.

2.5. Histochemical GUS Staining. GUS staining of rice calli and leaves was carried out using 5-bromo-4-chloro-3-indolyl glucuronide (X-Gluc) solution $\left(0.1 \mathrm{M} \mathrm{NaPO}_{4}, 0.1 \%\right.$ Triton X$100,0.5 \mathrm{mM}$ potassium ferrocyanide, $0.5 \mathrm{mM}$ potassium ferricyanide, $2 \mathrm{mM}$ X-Gluc, and $10 \mathrm{mM}$ EDTA pH 7.0) according to Jefferson et al. [20]. Bombarded calli and leaves were dipped in X-Gluc solution and incubated for 3-4 hrs at $37^{\circ} \mathrm{C}$. The experiment was repeated three times. 


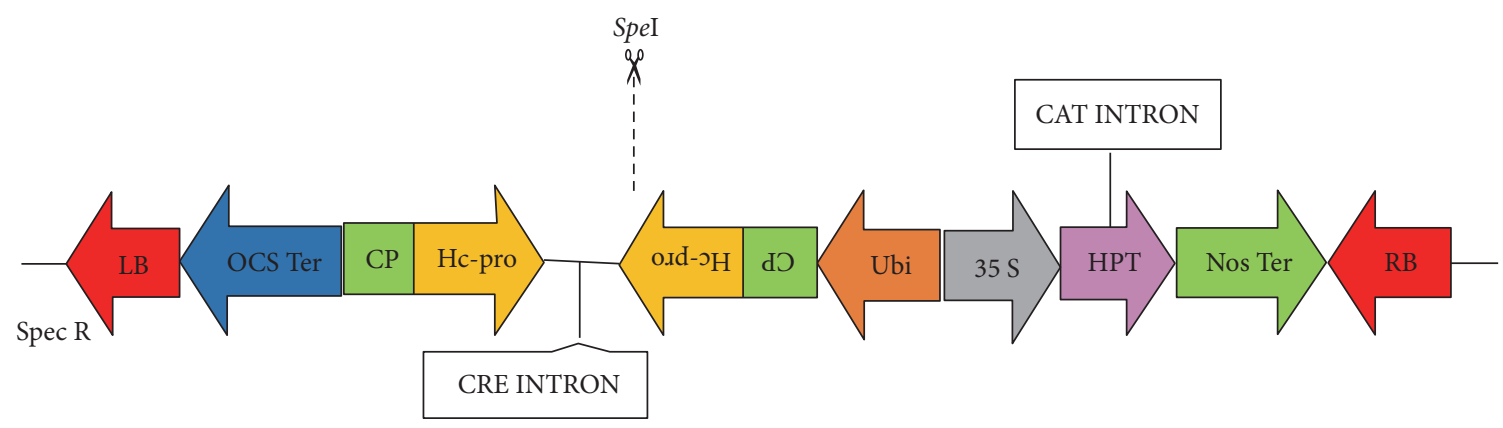

(a)

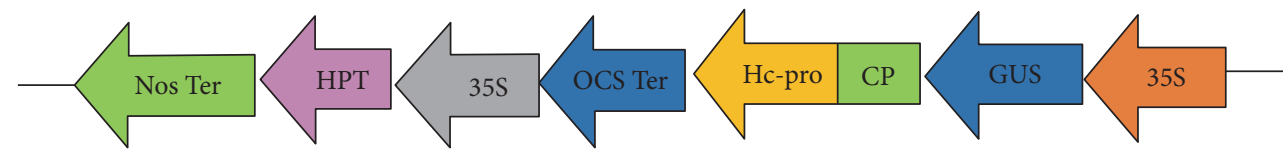

(b)

FIGURE 1: Schematic diagrams representing the chimeric CP:Hc-Pro hairpin RNA construct Ubi-hpCP:Hc-Pro (a) and the target reporter gene construct 35S-GUS:CP:Hc-Pro (b). 35S, Cauliflower mosaic virus 35S promoter; Ubi, maize ubiquitin promoter; HPT, hygromycin phosphate transferase gene; OCS Ter, Agrobacterium octopine synthase gene terminator sequence; Nos Ter, Agrobacterium nopaline synthase gene terminator; CAT INTRON, first intron of castor bean catalase-1 gene; CRE INTRON, the third intron of the Aegilops tauschii go35 NBS-LRR (go35) gene; SpeI, restriction site used for Southern blot analysis; LB, T-DNA left border; RB, right border.

2.6. RNA Extraction and Northern Blot Analysis. Total RNA was extracted from leaf of transgenic rice plants using TRIzol ${ }^{\circledR}$ Reagent (Thermo Fisher Scientific) following the manufacturer's recommendation.

For Northern blot analysis of target gene expression, total RNA (15 $\mu \mathrm{g})$ was separated in $1.3 \%$ formaldehydeagarose gel and blotted onto HyBond-N nylon membrane (GE Healthcare Amersham, Rydalmere, NSW, Australia).

${ }^{32} \mathrm{P}$-labelled antisense CP:Hc-Pro RNA probe was prepared by linearizing the CP:Hc-Pro plasmid in the $\mathrm{pENTR}^{\mathrm{TM}} / \mathrm{D}$ $\mathrm{TOPO}^{\circledR}$ vector with SpeI, followed by in vitro transcription using Sp6 RNA polymerase in the presence of ${ }^{32} \mathrm{P}$-UTP. Hybridization was performed as previously described [21].

For small interfering RNA (siRNA) detection, approximately $30 \mu \mathrm{g}$ total RNA was separated in $17 \%$ denaturing polyacrylamide gel. RNA was electroblotted to HyBond- $\mathrm{N}^{+}$ nylon membrane (GE Healthcare Amersham) using 0.5x TBE buffer. After UV cross-linking, the membrane was hybridized at $42^{\circ} \mathrm{C}$ with ${ }^{32} \mathrm{P}$ labeled antisense CP:Hc-Pro RNA probe [22]. Washing of hybridized membrane was carried out for three times at $42^{\circ} \mathrm{C}$ with $2 \mathrm{x}$ SSC containing $0.2 \%$ SDS. Hybridized membranes were visualized and recorded with phosphorimager (FLA-5000, Fuji Photo Film, Tokyo, Japan) after overnight exposure. The intensity of the 21-24 nt siRNA bands and the corresponding U6 RNA bands was determined three times against the background of the gel blots using the Multi Gauge Version 3.0 software (FUJIFILM). The average siRNA band intensity was then normalized against the average U6 RNA band intensity to generate the quantification data.

2.7. DNA Extraction, PCR Detection, and Southern Blot Analysis. Genomic DNA of rice plants was extracted from leaf tissue using CTAB protocol according to [23]. Forward
$\left(5^{\prime}\right.$-ATCTCACCGACTACAGCTTAG- $\left.3^{\prime}\right)$ and reverse $\left(5^{\prime}\right.$ GGTGTTACGTGTTTTTCATATGC- $3^{\prime}$ ) primers were used for PCR detection of the Ubi-hpRNA transgene using the following conditions: initial denaturation at $95^{\circ} \mathrm{C}$ for $5 \mathrm{~min}$, followed by 35 cycles of $95^{\circ} \mathrm{C}$ for $30 \mathrm{~s}, 54^{\circ} \mathrm{C}$ for $30 \mathrm{~s}$, and extension at $72^{\circ} \mathrm{C}$ for $1 \mathrm{~min}$, with a final extension at $72^{\circ} \mathrm{C}$ for 5 min.

For Southern blot analysis of transgene copy number, approximately $15 \mu \mathrm{g}$ of RNase A-treated rice genomic DNA was digested with SpeI and separated in 1\% agarose gel along with $1 \mathrm{~kb}$ plus DNA marker (Gene Ruler, Thermo Fisher Scientific, Walthan, MA) in 1x TBE buffer and transferred onto the Nylon- $\mathrm{N}^{+}$membrane (GE Healthcare Amersham). Probe was prepared by excising the CP:Hc-Pro fragment from the TOPO vector with SpeI and XhoI followed by gel purification. Purified fragment was labeled with ${ }^{32} \mathrm{P}$ radioactive dCTP using Deca label ${ }^{\mathrm{TM}}$ DNA labeling kit (Thermo Fisher Scientific) and hybridized with membrane at $65^{\circ} \mathrm{C}$ in hybridization buffer as previously described [24]. After hybridization, membranes were washed in 1x SSC for $20 \mathrm{~min}$ first at room temperature, followed by washes at $50^{\circ} \mathrm{C}$ and $60-65^{\circ} \mathrm{C}$. Blots were visualized and recorded by FLA-5000 phosphorimager (Fuji Photo Film) after overnight exposure.

2.8. Assay of Transgenic Rice Plants for SCMV Resistance. Transgenic rice plants at 5-10 leaf stage were inoculated with SCMV-strain A by rubbing carborundum-dusted leaves with extract of SCMV-infected sugarcane leaves in $0.1 \mathrm{M}$ sodium phosphate buffer ( $\mathrm{pH}$ 7.0). Infected leaves were harvested at 25 days after inoculation (DPI), and total RNA was extracted using TRIzol reagent (Thermo Fisher Scientific). The presence of SCMV was analyzed using RT-qPCR using 


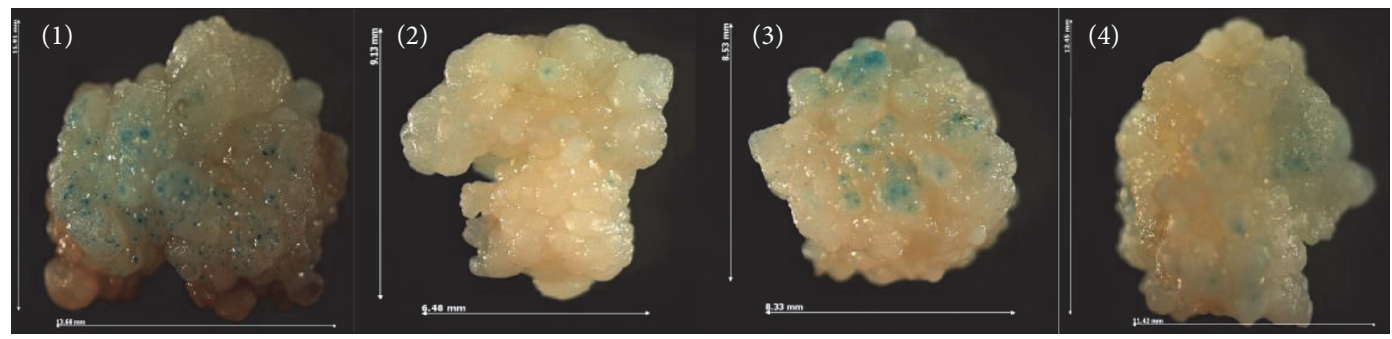

(a)

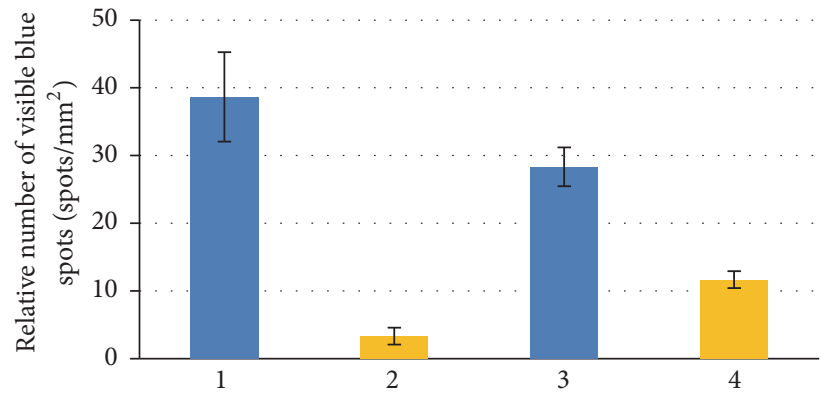

(b)

Figure 2: Transient expression assay to evaluate Ubi-hpCP:Hc-Pro construct in rice callus tissue. (a) X-glucuronide staining of rice callus tissue bombarded with (1) 35S-GUS:CP:Hc-Pro, (2) 35S-GUS:CP:Hc-Pro + Ubi-hpCP:Hc-Pro, (3) 35S-GUS:Y-Sat, and (4) 35S-GUS:Y-Sat + Ubi-hpCP:Hc-Pro constructs. Scale bar shows length and width of callus. (b) Histogram showing the average number of blue spots per square millimeter of bombarded rice callus tissue. Error bar represents standard deviation $(n=3)$.

a pair of $C P$ and $H c$-Pro specific primers: $5^{\prime}$-TTACAACGAAGATGTTTTCC-3' (CP-F), 5' -CTGAAATAGTAAATACGAGG-3' (CP-R) and $5^{\prime}$-CACAGAGCACACACCTACC$3^{\prime}$ (Hc-Pro-F), $5^{\prime}$-CCCAAATTCATCATCCGATAG-3' (HcPro-R). The rice $\beta$-tubulin gene (GenBank Accession number \# XM_015794238) was used as the internal reference for the RT-qPCR, using the following PCR primers: TUB-F $5^{\prime}$ GCTGACCACACCTAGCTTTG-3' and TUB-R 5'-AGGGAACCTTAGGCAGCATG-3'. RT-qPCR was performed in three technical replicates in the Rotor-Gene Real-Time PCR System (Qiagen, Hilden, Germany) using Platinum Taq polymerase (Invitrogen, Thermo Fisher Scientific) and SYBR green.

\section{Results}

3.1. Development of Ubi-hpCP:Hc-Pro and 35S-GUS:CP:HcPro Constructs. Selection of a suitable target gene sequence can be important for successful knockdown by RNA interference-based approaches. The hpRNA construct was developed targeting $\mathrm{CP}$ and Hc-Pro genes to interfere with the encapsidation, vector mediated virus transmission, and RNA silencing suppressor simultaneously. The consensus sequences of $240 \mathrm{bp}$ each of CP gene sequences (between 602 and $841 \mathrm{nt}$ ) covering the conserved DAG motif near $\mathrm{N}$-terminus and Hc-Pro gene sequence (between 121 and $359 \mathrm{nt}$ ) containing the N-terminus KITC motif were selected through multiple sequence alignments (data not shown). The selected $C P$ and Hc-Pro gene sequences were subsequently joined together to form a chimera sequence (CP:Hc-Pro) which was arranged in an inverted repeat configuration in the hpRNA construct and successfully cloned under the maize ubiquitin promoter (Ubi), to develop Ubi-hpCP:HcPro expression construct (Figure 1(a)). The chimera sequence was fused transcriptionally with GUS reporter gene and subcloned into pART7 [18] between CaMV 35S promoter and the octopine synthase (OCS) terminator, to develop the $35 \mathrm{~S}$ GUS:CP:Hc-Pro target construct for detecting the hpRNA silencing efficiency (Figure 1(b)).

\subsection{The Ubi-hpCP:Hc-Pro Construct Induces Efficient Target} Gene Silencing in Rice Callus Tissue. The efficacy of the UbihpCP:Hc-Pro expression construct was validated through a transient expression assay by biolistic delivery of both the targeted GUS reporter gene construct (35S-GUS:CP:Hc-Pro) and the Ubi-hpCP:Hc-Pro construct into rice callus tissue. Another GUS construct (35S-GUS:Y-Sat), with a $3^{\prime}$ fusion of the Cucumber mosaic virus Y-satellite RNA sequence [25], was included as a control target. Histochemical staining of the bombarded rice calli showed a clear reduction of blue spots that represented GUS expressing cells, upon cobombardment with the Ubi-hpCP:Hc-Pro construct (Figure 2(a)). On average, 38 blue spots per square millimetre area were observed in rice calli bombarded with $35 \mathrm{~S}$ GUS:CP:Hc-Pro alone, and this was in contrast to only about 3 blue spots in calli cobombarded with 35S-GUS:CP:Hc-Pro and Ubi-hpCP:Hc-Pro (Figure 2(b)). This result indicated that the Ubi-hpCP:Hc-Pro construct, comprising the $C P$ and Hc-Pro sequences, induced sequence-specific suppression of gene expression against the CP:Hc-Pro sequence in the 35S-GUS:CP:Hc-Pro target construct, resulting in 
downregulation of GUS expression and hence diminished number of blue spots. It is worth noting that cobombardment with the Ubi-hpCP:Hc-Pro construct also reduced the number of blue spots from the expression of the 35SGUS:Y-Sat construct, but at a lesser degree from an average of 27 per square millimetre to 12 . It is unclear why this reduction occurred but it could be due to the sharing of the OCS terminator sequence between the hpRNA and the 35S-GUS:Y-Sat constructs that could induce transgene cosuppression.

\subsection{Transgenic Rice Plants Containing Ubi-hpCP:Hc-Pro} Accumulate siRNAs. The rice plant, a model monocotyledonous species, was used as a surrogate of sugarcane because of its transformation efficiency, compared to the other cereal crops, such as wheat, maize, and barley and the conservation of gene sequences [26-30]. The Ubi-hpCP:Hc-Pro and the empty vector control (VC) constructs were transformed into rice using Agrobacterium-mediated methods, generating the six independent transgenic lines, respectively. One empty vector control and five Ubi-hpCP:Hc-Pro primary transgenic lines $\left(\mathrm{T}_{0}\right)$ were analyzed for transgene copy number by Southern blot hybridization and for the accumulation of siRNAs by Northern blot hybridization. Empty vector control line showed no CP:Hc-Pro hybridization signals on Southern blot while lines \#2, \#3, and \#4 showed a hybridizing band indicating that each of these lines may contain a single copy of transgene. The size of the hybridizing band for lines \#2, $\# 3$, and \#4 appears to be the same ( 12 kb), which seems to indicate that they came from a single transgenic event. This is despite the fact that plants were derived from different callus lines. Lines \#1 and \#5 contained multiple copies as indicated by the multiple hybridizing bands on the Southern blot (Figure 3).

Small interfering RNAs (siRNAs) 21-24 nts in size, corresponding to the CP:Hc-Pro sequence of the Ubi-hpCP:HcPro, were readily detected by Northern blot analysis in all the five independent lines (Figure 4(a)), indicating that the Ubi-hpCP:Hc-Pro was expressed and the resulting hpRNA processed by Dicer (DCLs) enzyme which recognizes and cleaves the dsRNA into siRNAs. The size distribution of the siRNA was consistent with that of hpRNA-derived siRNAs in Arabidopsis (Arabidopsis thaliana), consisting of 21, 22, and 24 nt size classes [31], (although the 21 and $22 \mathrm{nt}$ species were not clearly separated) (Figure 4(a)). This indicates that the CP:Hc-Pro hpRNA was processed in transgenic rice by DCL4, DCL2, and DCL3, respectively, corresponding to the pattern observed in Arabidopsis [32]. In a previous report, DCL4 dependent 21 nt siRNAs were the predominant species of siRNAs derived from the SCMV [33]. Moreover, DCL2 and DCL4 function redundantly in maize and Arabidopsis plants to produce higher level of $21 \mathrm{nt}$ and $22 \mathrm{nt}$ siRNAs $[33,34]$. It is noteworthy that in the different transgenic lines the siRNA hybridization signals showed different intensity, indicating a variable level of siRNA in these lines (Figure 4(b)). Interestingly, the high-copy-number transgenic line \#5 did not accumulate more siRNAs; instead, it appears to generate slightly less siRNAs, suggesting that the multiple

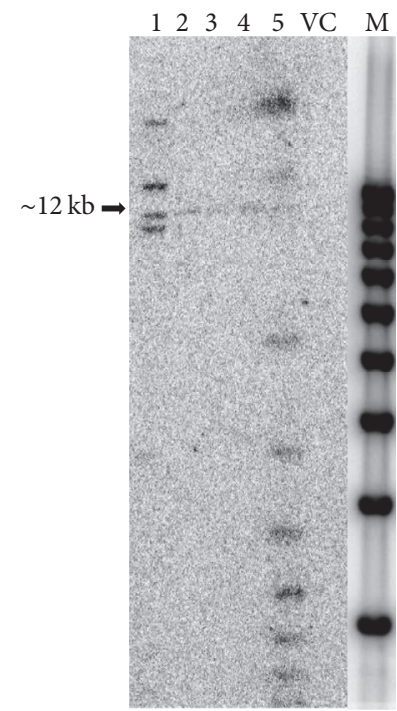

FIGURE 3: Southern blot analysis of $\mathrm{T}_{0}$ transgenic rice plants. DNA extracted from transgenic rice leaves was digested with SpeI restriction enzyme, separated in $0.8 \%$ agarose gel, transferred to HyBond- $\mathrm{N}^{+}$Nylon membrane, and hybridized with radioactive labeled CP:Hc-Pro DNA probe. Numbers 1-5 indicate five UbihpCP:Hc-Pro transgenic lines. VC, transgenic rice plants containing the empty vector control transgene. M, DNA marker ( $1 \mathrm{~kb}$ plus Gene Ruler). The arrow indicates the single transgene band in lines 2, 3, and 4 .

copy transgene insertion may have resulted in transcription repression of the hpRNA transgene [35].

3.4. The Ubi-hpCP:Hc-Pro Transgene Confers Silencing to a Biolistically Delivered Target Gene. The efficacy of UbihpCP:Hc-Pro transgene against the target viral sequences was shown in a transient expression assay by delivering the $35 \mathrm{~S}$ GUS:CP:Hc-Pro target construct into transgenic rice leaves using particle bombardment. Strong GUS expression was observed in leaf tissue of the empty vector control transgene but not in the leaves of the Ubi-hpCP:Hc-Pro transgenic plants (three replicates of all transgenic lines), indicating that siRNAs derived from the Ubi-hpCP:Hc-Pro induced silencing against the 35S-GUS:CP:Hc-Pro target construct by targeting CP:Hc-Pro fusion sequence (Figure S2).

3.5. Ubi-hpCP:Hc-Pro Transgene Stably Inherited in $T_{1}$ Generation and Confers Resistance against SCMV. To investigate if the Ubi-hpCP:Hc-Pro transgenic plants were resistant to SCMV infection, seeds were collected from lines \#2, \#3, \#4, and \#5 primary transgenic plants (line \#1 did not produce seeds) from which $\mathrm{T}_{1}$ plants were established and assayed for transgene expression and SCMV resistance. The Ubi-hpCP:Hc-Pro transgene expression, as indicated by the hybridizing signals of the unprocessed CP:Hc-Pro hpRNA on the Northern blot, was clearly detected in the $T_{1}$ progeny of the single-copy lines \#2, $\# 3$, and \#4, indicating stable inheritance from the primary transgenic plants (Figure 5(a)). However, for line \#5, the highest-copy-number transgenic line, three of the 


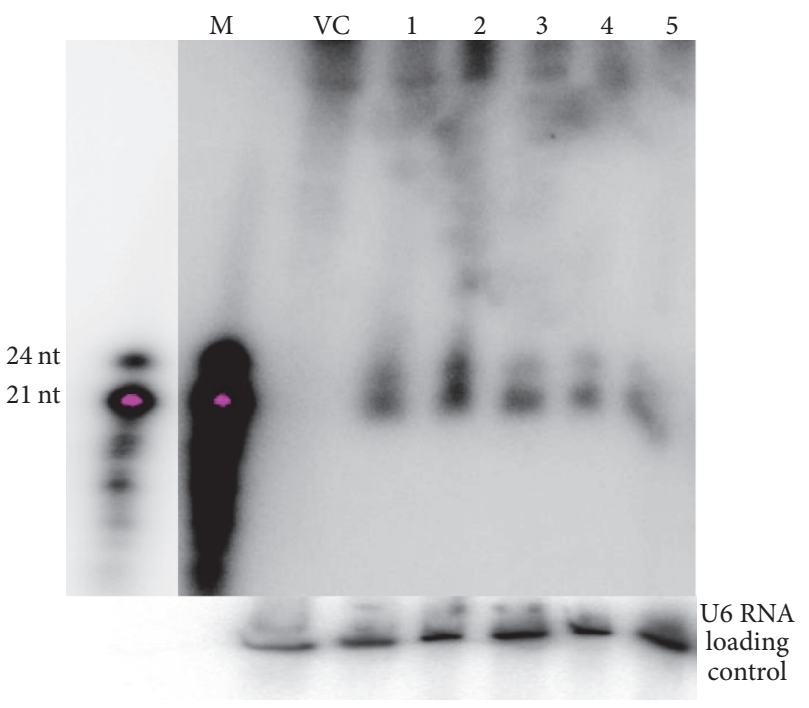

(a)

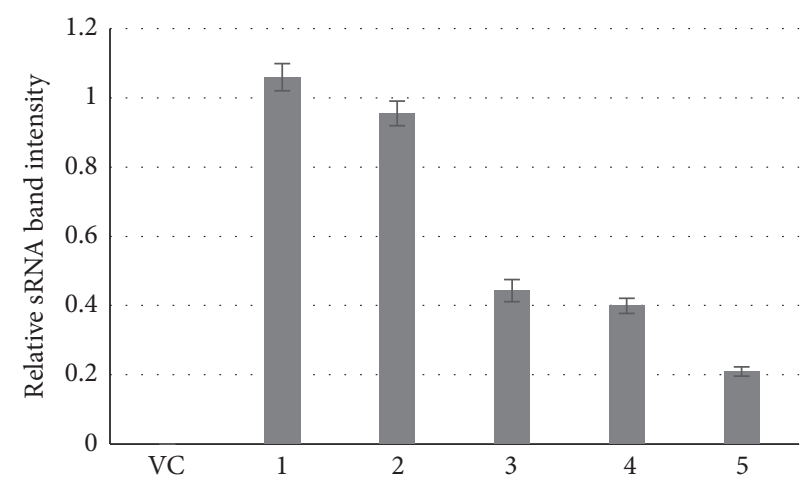

(b)

FIgURE 4: $\mathrm{T}_{0}$ transgenic rice plants express 21-24 nt siRNAs. (a) Northern blot hybridization for the detection of siRNA from transgenic rice lines. Twenty-five $\mu \mathrm{g}$ of total RNA extracted from leaves of transgenic plants was hybridized with antisense CP:Hc-Pro RNA probe (the upper panel). The U6 small nuclear RNA was hybridized as a loading control (the lower panel). Lane M represents the 21-24 nt radiolabeled RNA size marker, with a less-exposed picture given on the left to clearly identify the 21 and $24 \mathrm{nt}$ bands. VC is transgenic rice plants containing the empty vector control transgene while, Lanes 1-5 are the same five Ubi-hpCP:Hc-Pro transgenic rice lines shown in Figure 3. (b) Histogram representing the relative intensity of the sRNA band on the Northern blot above.

four $\mathrm{T}_{1}$ plants analyzed showed no Ubi-hpCP:Hc-Pro signal, which may indicate the transcriptional suppression of the Ubi-hpCP:Hc-Pro transgene in these individuals of high copy number transgene insertion. It is also noticeable that plant \#5 from line 3 also showed no Ubi-hpCP:Hc-Pro hybridization signals (Figure 5(a)).

To examine if the Ubi-hpCP:Hc-Pro transgene could confer resistance to SCMV infection, a number of $\mathrm{T}_{1}$ plants of line \#3, shown to express high levels of Ubi-hpRNA in the Northern blot (Figure 5(a)), together with $\mathrm{T}_{1}$ plants of an empty vector control line, were inoculated with SCMV. The $12 \mathrm{~T}_{1}$ Ubi-hpCP:Hc-Pro plants showed visibly higher growth vigor than the 12 empty vector control plants at 6 weeks after inoculation, implying resistance to SCMV (strain A, a mild strain of SCMV) infection (Figure 5(b)). SCMV strain A infection studies typically showed stunted growth with mild mosaic pattern without necrosis in its natural hosts that are sugarcane, maize, and sorghum [36]. In the present study, no clear mosaic symptoms were observed in the SCMV-inoculated plants possibly because rice is not the natural host of SCMV and very mild mosaic symptoms were reported through artificial inoculation of SCMV [36]. To confirm the successful SCMV resistance, RNA was extracted from a subset of the inoculated plants and analyzed for the presence of SCMV using RT-qPCR with $\mathrm{CP}$ and Hc-Pro specific primers. The empty vector control plants showed high levels of SCMV CP and Hc-Pro RNA (Figure 5(c)). In contrast, no significant amount of SCMV RNA was detectable in all five $\mathrm{T}_{1}$ transgenic plants analyzed, indicating that the Ubi-hpCP:Hc-Pro transgenic plants (from line \#3) were highly resistant to SCMV infection. Both the $C P$ and
Hc-Pro target sequences were similarly silenced in the SCMVinfected Ubi-hpCP:Hc-Pro transgenic plants (Figure 5(c)), indicating that the transgene was effective against both genes.

\section{Discussion}

SCMV is an agronomically important Potyvirus infecting a variety of monocotyledonous species including important crop plants such as maize and sugarcane. It is transmitted in nature by five different insect vectors: Aphis craccivora, Aphis gossypii, Macrosiphum euphorbiae, Rhopalosiphum maidis, and Rhopalosiphum padi [37]. Outbreak of this virus often causes severe damage to crops leading to high cost to crop yields in more than 70 countries [38]. According to the survey conducted between 2008 and 2010 in Pakistan, the highest incidence of virus infection in sugarcane fields reached up to $38 \%$ in the Punjab province of Pakistan during the year 2010 [39].

Because of the potential damage SCMV could do to the agronomically important monocotyledonous crops, many efforts have been made to control the spread and multiplication of this virus. It was reported that protection against severe SCMV strains was achieved in a number of different crops by inoculating plants with mild virus strain through the mechanism of viral cross protection $[40,41]$. Attempts have also been made in the selection and breeding for naturally occurring resistant crop varieties for controlling SCMV. For example, a European maize inbred line was found to be completely resistant to SCMV and Maize dwarf mosaic virus in green house and field tests [42]. As a number of plant 


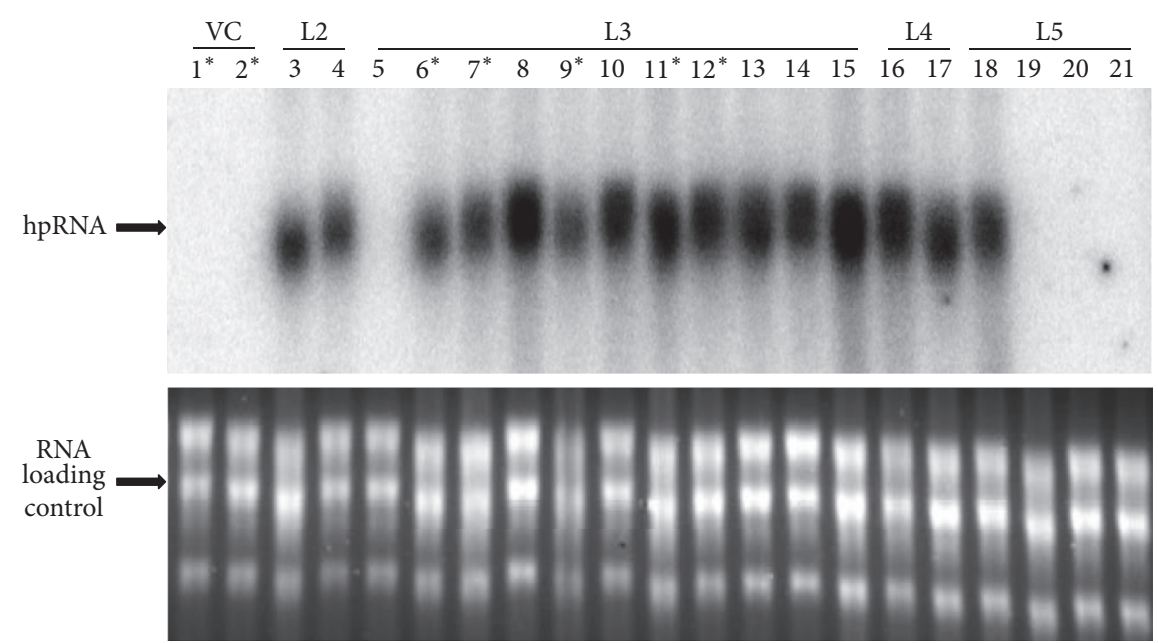

(a)

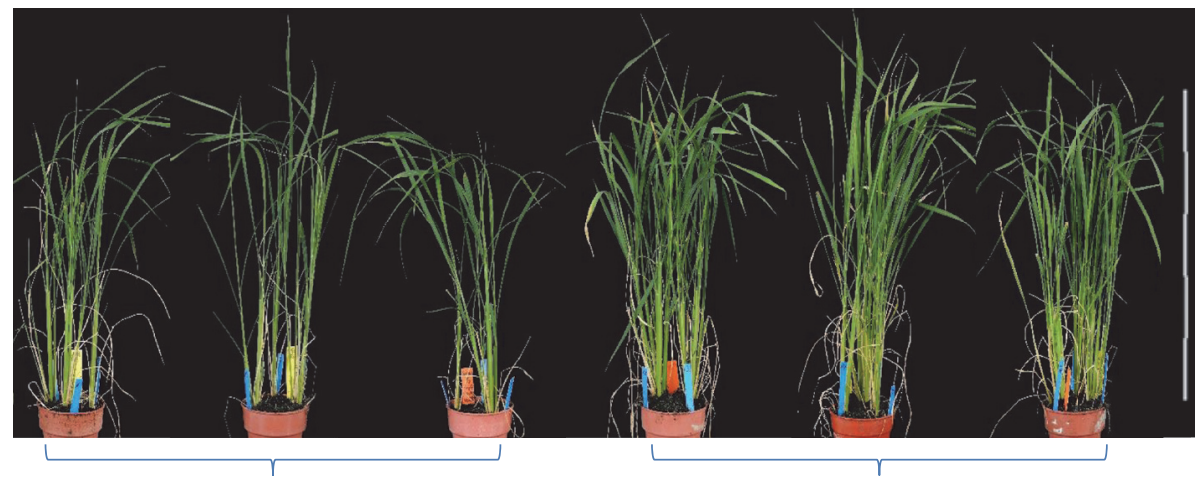

$12 \mathrm{~T} 1$ plants of VC line

$12 \mathrm{~T} 1$ plants of $\mathrm{L} 3$

(b)
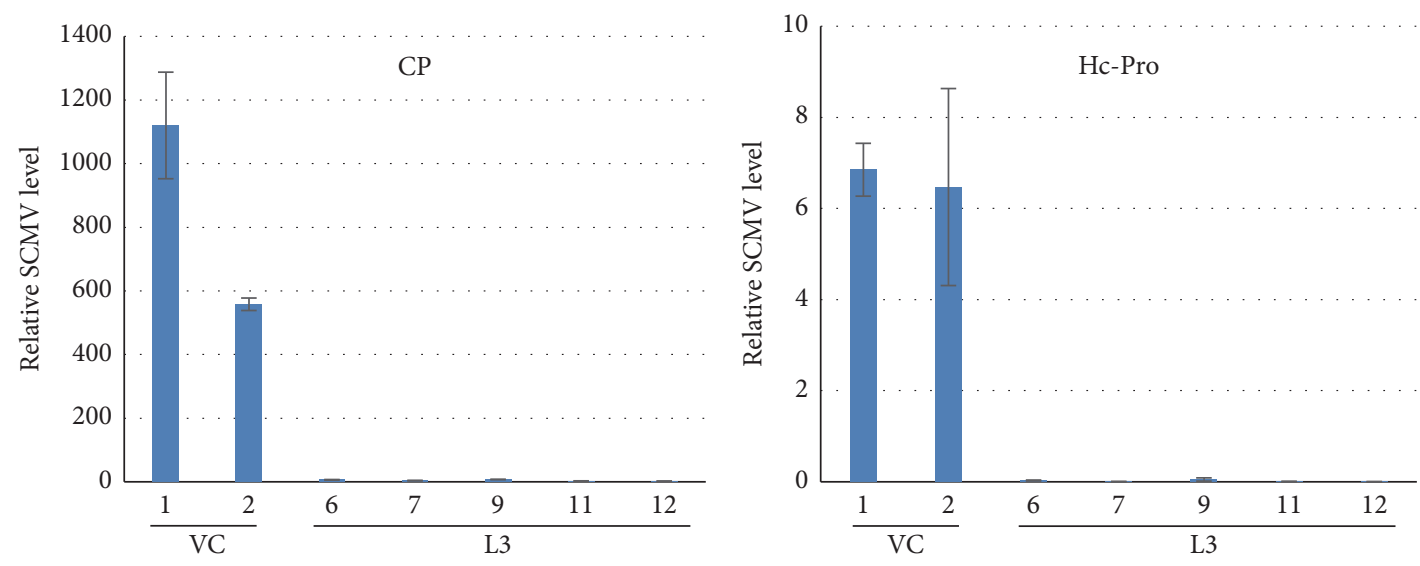

(c)

FIGURE 5: Ubi-hpCP:Hc-Pro transgene expression and SCMV resistance analysis in $\mathrm{T}_{1}$ generation. (a) Northern blot hybridization of $\mathrm{T}_{1}$ progeny of four independent Ubi-hpCP:Hc-Pro lines (\#2, \#3, \#4, and \#5) and an empty vector control line (VC) using ${ }^{32} \mathrm{P}$-labelled antisense CP:Hc-Pro RNA as probe, which shows Ubi-hpCP:Hc-Pro expression from \#2, \#3 (except for one plant), \#4, and one of \#5 plants, but not from the vector control and the other three \#5 plants. Lower panel shows RNA loading control. Asterisks indicate plants analysed in (c). (b) Vector control and Ubi-hpCP:Hc-Pro transgene $\mathrm{T}_{1}$ transgenic rice plants inoculated six weeks before with SCMV. (c) Quantitative real-time RT-PCR analysis of SCMV accumulation in infected VC and hpRNA transgenic T1 plants using CP (left) or Hc-Pro (right) primers. 
viruses such closteroviruses, comoviruses, tymoviruses, and nepoviruses utilize cysteine proteinases in their polyprotein processing, a cysteine proteinase inhibitor, cystatin, has been transgenically expressed in tobacco to control the multiplication of these viruses [43], which could be potentially used to control SCMV. However, an effective method for controlling SCMV and SCMV-caused diseases has so far not been found.

RNA silencing-based approaches, especially the long hpRNA technology, has been successfully used to engineer plant resistance against viruses, including agronomically important potyviruses, such as Wheat streak mosaic virus, Potato virus Y, and Plum pox virus [44, 45]. Various regions of the potyviral genome, such as those encoding the NIa protease and Hc-Pro, have been selected as the target sequences for the hpRNA transgenes in such experiments. For the SCMV, attempts were also made to transform maize with an hpRNA construct targeting the $C P$ gene, generating transgenic plants with variable levels of SCMV resistance [46]. Similarly, SrMV resistant sugarcane transgenic lines were generated using hpRNA transgene targeting $C P$ region showing different levels of virus resistance [47]. Thus, hpRNA transgenes provide a potential opportunity for developing SCMV resistance in important crops such as sugarcane.

While targeting a single viral gene by hpRNA transgenes has proved to be successful, we chose a strategy of simultaneously targeting two essential SCMV genes, $C P$ and Hc-Pro. While $C P$ is essential for viral encapsidation and transmission, Hc-Pro has multiple functions including the function of countering RNA silencing. It was anticipated that simultaneous silencing of the CP and the Hc-Pro genes would both cause direct downregulation of viral RNAs and enhance host antiviral silencing activity, therefore resulting in robust virus resistance in the transgenic plants. We selected 240 bp segments from each of the $C P$ and $\mathrm{Hc}$-Pro genes that correspond to conserved functional motifs, with the purpose of achieving broad resistance against the various SCMV virus isolates. In addition, the maize ubiquitin promoter was chosen to drive the expression of the Ubi-hpCP:Hc-Pro transgene, which has been shown to be suitable for high levels of transgene expression in rice and other monocot species [48]. Finally, in the Ubi-hpCP:Hc-Pro construct the sense and antisense CP:Hc-Pro sequences are separated by a spliceable intron, which is suggested to enhance the efficacy of hpRNA transgenes [49].

The efficacy of the Ubi-hpCP:Hc-Pro transgene was first demonstrated by showing that it could induce efficient silencing against the 35S-GUS:CP:Hc-Pro fusion transgene when transiently coexpressed in rice callus tissue using particle bombardment. When stably transformed into rice plants, this Ubi-hpCP:Hc-Pro transgene gave rise to siRNAs across the independent transgenic lines, which conferred silencing against the biolistically delivered 35S-GUS:CP:HcPro fusion transgene. The size distribution of the siRNAs is consistent with the typical pattern of hpRNA-derived siRNAs [31], with the 21-22 nt siRNAs being more abundant than the $24 \mathrm{nt}$ species. Interestingly, the abundance of siRNAs showed no positive correlation with the copy number of UbihpCP:Hc-Pro transgene insertion, and it appeared that the higher the copy number is, the less the siRNA accumulates.
Analysis of Ubi-hpCP:Hc-Pro transcript accumulation in the subsequent $T_{1}$ population further suggested that the UbihpCP:Hc-Pro transgene is subject to transcriptional silencing when the number of transgene insertions is very high, which is consistent with previous reports [50]. It was previously reported that high copy number or polyploid progenitor line undergoes partial or complete transgene silencing which is caused by transacting silencing of active and inactive epialleles [51]. This suggests that for achieving stable virusresistance, transgenic plants with a simple transgene insertion pattern should be selected.

Virus infection assays of the single-copy transgenic line expressing high levels of the Ubi-hpCP:Hc-Pro showed that the transgenic plants are highly resistant to SCMV, which displayed a more vigorous growth than vector control transgenic plants, with almost no detectable SCMV RNA in contrast to SCMV RNA in the vector control plants.

In conclusion, we demonstrate here that high-level SCMV resistance can be achieved using an hpRNA transgene targeting two essential viral genes at the same time. While this strategy has not been tested in sugarcane it is anticipated that the Ubi-hpCP:Hc-Pro construct simultaneously targeting the downregulation of both $C P$ and Hc-Pro expression in SCMV will confer SCMV resistance when transformed into sugarcane.

\section{Competing Interests}

The authors declare no conflict of interests.

\section{Authors' Contributions}

Muhammad Tahir, Ming-Bo Wang, and Qing Liu conceived and designed the experiments. Sehrish Akbar, Ming-Bo Wang, and Qing Liu performed the experiments. All the authors contributed to the writing of the manuscript and agree with its content.

\section{Acknowledgments}

The authors would like to thank Sugar Research Australia for providing the SCMV-infected sugarcane samples. This work was funded by Higher Education Commission (HEC) Pakistan. Excellent technical assistance from Dr. Dawar Hussain, Neil Smith, and Luch Hac is also acknowledged.

\section{References}

[1] S. Anwar, "Impact of sugarcane disease on cane and sugar yield," in Proceedings of the 3rd Agricultural Biotechnology Workshop, Agricultural Biotechnology Research Institute, Faisalabad, Pakistan, 2005.

[2] J. Verchot, E. V. Koonin, and J. C. Carrington, “The $35 \mathrm{kDa}$ protein from the $\mathrm{N}$-terminus of the potyviral polyprotein functions as a third virus-encoded proteinase," Virology, vol. 185, no. 2, pp. 527-535, 1991.

[3] J. L. Riechmann, S. Lain, and J. A. Garcia, "Highlights and prospects of potyvirus molecular biology," Journal of General Virology, vol. 73, no. 1, pp. 1-16, 1992. 
[4] D. D. Shukla and C. W. Ward, "Identification and classification of potyviruses on the basis of coat protein sequence data and serology," Archives of Virology, vol. 106, no. 3-4, pp. 171-200, 1989.

[5] K. D. Kasschau and J. C. Carrington, "Long-distance movement and replication maintenance functions correlate with silencing suppression activity of potyviral HC-Pro," Virology, vol. 285, no. 1, pp. 71-81, 2001.

[6] K. D. Kasschau and J. C. Carrington, "A counterdefensive strategy of plant viruses: suppression of posttranscriptional gene silencing," Cell, vol. 95, no. 4, pp. 461-470, 1998.

[7] C. D. Atreya and T. P. Pirone, "Mutational analysis of the helper component-proteinase gene of a potyvirus: effects of amino acid substitutions, deletions, and gene replacement on virulence and aphid transmissibility," Proceedings of the National Academy of Sciences of the United States of America, vol. 90, no. 24, pp. 1191911923, 1993.

[8] D. A. Govier and B. Kassanis, "Evidence that a component other than the virus particle is needed for aphid transmission of potato virus Y," Virology, vol. 57, no. 1, pp. 285-286, 1974.

[9] P. A. Joyce, R. B. McQualter, J. A. Handley, J. L. Dale, R. M. Harding, and G. R. Smith, "Transgenic sugarcane resistant to Sugarcane mosaic virus," Proceedings of the Australian Society of Sugar Cane Technology, vol. 20, pp. 204-210, 1998.

[10] I. L. Ingelbrecht, J. E. Irvine, and T. E. Mirkov, "Posttranscriptional gene silencing in transgenic sugarcane. Dissection of homology-dependent virus resistance in a monocot that has a complex polyploid genome," Plant Physiology, vol. 119, no. 4, pp. 1187-1197, 1999.

[11] N. Baumberger and D. C. Baulcombe, "Arabidopsis ARGONAUTE1 is an RNA Slicer that selectively recruits microRNAs and short interfering RNAs," Proceedings of the National Academy of Sciences of the United States of America, vol. 102, no. 33, pp. 11928-11933, 2005.

[12] O. Voinnet, "Induction and suppression of RNA silencing: insights from viral infections," Nature Reviews Genetics, vol. 6, no. 3, pp. 206-220, 2005.

[13] Y. Tomari and P. D. Zamore, "Perspective: machines for RNAi," Genes and Development, vol. 19, no. 5, pp. 517-529, 2005.

[14] J. Guo, S. Gao, Q. Lin, H. Wang, Y. Que, and L. Xu, "Transgenic sugarcane resistant to Sorghum mosaic virus based on coat protein gene silencing by RNA interference," BioMed Research International, vol. 2015, Article ID 861907, 9 pages, 2015.

[15] F. Wang, W. Li, J. Zhu et al., "Hairpin RNA Targeting Multiple Viral Genes Confers Strong Resistance to Rice Black-Streaked Dwarf Virus," International Journal of Molecular Sciences, vol. 17, no. 5, article 705, 2016.

[16] J. D. Thompson, D. G. Higgins, and T. J. Gibson, "CLUSTAL W: improving the sensitivity of progressive multiple sequence alignment through sequence weighting, position-specific gap penalties and weight matrix choice," Nucleic Acids Research, vol. 22, no. 22, pp. 4673-4680, 1994.

[17] M.-B. Wang, Z.-Y. Li, P. R. Matthews, N. M. Upadhyaya, and P. M. Waterhouse, "Improved vectors for Agrobacterium tumefaciens-mediated transformation of monocot plants," Acta Horticulturae, vol. 461, pp. 401-407, 1998.

[18] A. P. Gleave, "A versatile binary vector system with a T-DNA organisational structure conducive to efficient integration of cloned DNA into the plant genome," Plant Molecular Biology, vol. 20, no. 6, pp. 1203-1207, 1992.

[19] Y. Hiei, S. Ohta, T. Komari, and T. Kumashiro, "Efficient transformation of rice (Oryza sativa L.) mediated by agrobacterium and sequence analysis of the boundaries of the T-DNA," Plant Journal, vol. 6, no. 2, pp. 271-282, 1994.

[20] R. A. Jefferson, T. A. Kavanagh, and M. W. Bevan, "GUS fusions: beta-glucuronidase as a sensitive and versatile gene fusion marker in higher plants," EMBO Journal, vol. 6, no. 13, pp. 39013907, 1987.

[21] M.-B. Wang, S. V. Wesley, E. J. Finnegan, N. A. Smith, and P. $\mathrm{M}$. Waterhouse, "Replicating satellite RNA induces sequencespecific DNA methylation and truncated transcripts in plants," RNA, vol. 7, no. 1, pp. 16-28, 2001.

[22] M.-B. Wang, C. A. Helliwell, L.-M. Wu, P. M. Waterhouse, W. J. Peacock, and E. S. Dennis, "Hairpin RNAs derived from RNA polymerase II and polymerase III promoter-directed transgenes are processed differently in plants," RNA, vol. 14, no. 5, pp. 903913, 2008.

[23] J. Draper and R. Scott, "The isolation of plant nucleic acids," in Plant Genetic Transformation and Gene Expression: A Laboratory Manual, J. Draper, R. Scott, P. Armitage, and R. Walden, Eds., pp. 199-236, Alden Press, Oxford, UK, 1988.

[24] S. Rapson, M. Wu, S. Okada et al., "A case study on the genetic origin of the high oleic acid trait through FAD2-1 DNA sequence variation in safflower (Carthamus tinctorius L.)," Frontiers in Plant Science, vol. 6, article 691, 2015.

[25] K. Zahid, J.-H. Zhao, N. A. Smith et al., "Nicotiana small RNA sequences support a host genome origin of cucumber mosaic virus satellite RNA," PLoS Genetics, vol. 11, no. 1, Article ID e1004906, 2015.

[26] K. Fukui and K. Iijima, "Somatic chromosome map of rice by imaging methods," Theoretical and Applied Genetics, vol. 81, no. 5, pp. 589-596, 1991.

[27] Y. Hiei, T. Komari, and T. Kubo, "Transformation of rice mediated by Agrobacterium tumefaciens," Plant Molecular Biology, vol. 35, no. 1, pp. 205-218, 1997.

[28] S. Ahn, J. A. Anderson, M. E. Sorrells, and S. D. Tanksley, "Homoeologous relationships of rice, wheat and maize chromosomes," MGG Molecular \& General Genetics, vol. 241, no. 5-6, pp. 483-490, 1993.

[29] K. M. Devos and M. D. Gale, "Comparative genetics in the grasses," Plant Molecular Biology, vol. 35, no. 1, pp. 3-15, 1997.

[30] K. M. Devos, T. S. Pittaway, A. Reynolds, and M. D. Gale, "Comparative mapping reveals a complex relationship between the pearl millet genome and those of foxtail millet and rice," Theoretical and Applied Genetics, vol. 100, no. 2, pp. 190-198, 2000.

[31] A. F. Fusaro, L. Matthew, N. A. Smith et al., "RNA interferenceinducing hairpin RNAs in plants act through the viral defence pathway," EMBO Reports, vol. 7, no. 11, pp. 1168-1175, 2006.

[32] Z. Xie, L. K. Johansen, A. M. Gustafson et al., "Genetic and functional diversification of small RNA pathways in plants," PLOS Biology, vol. 2, no. 5, article e104, 2004.

[33] Z. Xia, J. Peng, Y. Li et al., "Characterization of small interfering rnas derived from Sugarcane mosaic virus in infected maize plants by deep sequencing," PLOS ONE, vol. 9, no. 5, Article ID e97013, 2014.

[34] H. Garcia-Ruiz, A. Takeda, E. J. Chapman et al., “Arabidopsis RNA-dependent RNA polymerases and dicer-like proteins in antiviral defense and small interfering RNA biogenesis during Turnip mosaic virus infection," Plant Cell, vol. 22, no. 2, pp. 481496, 2010.

[35] M.-B. Wang and P. M. Waterhouse, "High-efficiency silencing of a $\beta$-glucuronidase gene in rice is correlated with repetitive 
transgene structure but is independent of DNA methylation," Plant Molecular Biology, vol. 43, no. 1, pp. 67-82, 2000.

[36] E. V. Abbott and R. L. Tippett, "Strains of Sugarcane mosaic virus," Technical Bulletin US Department of Agriculture, vol. 48, p. $443,1964$.

[37] D. S. Teakle and N. E. Grylls, "Four strains of sugarcane mosaic virus infecting cereals and other grasses in Australia," Australian Journal of Agricultural Research, vol. 24, no. 4, pp. 465-477, 1973.

[38] J. S. Hall, B. Adams, T. J. Parsons, R. French, L. C. Lane, and S. G. Jensen, "Molecular cloning, sequencing, and phylogenetic relationships of a new Potyvirus: Sugarcane streak mosaic virus, and a reevaluation of the classification of the Potyviridae," Molecular Phylogenetics and Evolution, vol. 10, no. 3, pp. 323332, 1998.

[39] T. Yasmin, S. Iqbal, A. Farooq, M. Zubair, and A. Riaz, "Prevalence, distribution and incidence of major sugarcane infecting viruses in NWFP and Punjab," Pakistan Journal of Phytopathology, vol. 23, no. 1, pp. 24-30, 2011.

[40] S. Pennazio, P. Roggero, and M. Conti, "A history of plant virology. Cross protection," New Microbiologica, vol. 24, no. 1, pp. 99-114, 2001.

[41] C. Zhou and Y. Zhou, "Strategies for viral cross protection in plants," Methods in Molecular Biology, vol. 894, pp. 69-81, 2012.

[42] M. D. McMullen, "The linkage of molecular markers to a gene controlling the symptom response in maize to Maize dwarf mosaic virus," Molecular Plant-Microbe Interactions, vol. 2, no. 6, pp. 309-314, 1989.

[43] R. Gutierrez-Campos, J. A. Torres-Acosta, L. J. SaucedoArias, and M. A. Gomez-Lim, "The use of cysteine proteinase inhibitors to engineer resistance against potyviruses in transgenic tobacco plants," Nature Biotechnology, vol. 17, no. 12, pp. 1223-1226, 1999.

[44] M. Fahim, A. A. Millar, C. C. Wood, and P. J. Larkin, "Resistance to Wheat streak mosaic virus generated by expression of an artificial polycistronic microRNA in wheat," Plant Biotechnology Journal, vol. 10, no. 2, pp. 150-163, 2012.

[45] D. Leibman, D. Wolf, V. Saharan et al., "A high level of transgenic viral small RNA is associated with broad Potyvirus resistance in cucurbits," Molecular Plant-Microbe Interactions, vol. 24, no. 10, pp. 1220-1238, 2011.

[46] E. Di Nicola-Negri, M. Tavazza, L. Salandri, and V. Ilardi, "Silencing of Plum pox virus 5'UTR/P1 sequence confers resistance to a wide range of PPV strains," Plant Cell Reports, vol. 29, no. 12, pp. 1435-1444, 2010.

[47] D. Gan, F. Ding, D. Zhuang et al., "Application of RNA interference methodology to investigate and develop SCMV resistance in maize," Journal of Genetics, vol. 93, no. 2, pp. 305311, 2014.

[48] M.-J. Cornejo, D. Luth, K. M. Blankenship, O. D. Anderson, and A. E. Blechl, "Activity of a maize ubiquitin promoter in transgenic rice," Plant Molecular Biology, vol. 23, no. 3, pp. 567$581,1993$.

[49] N. A. Smith, S. P. Singh, M.-B. Wang, P. A. Stoutjesdijk, A. G. Green, and P. M. Waterhouse, "Gene expression: total silencing by intron-spliced hairpin RNAs," Nature, vol. 407, no. 6802, pp. 319-320, 2000.

[50] M. Fagard and H. Vaucheret, "(Trans)gene silencing in plants: how many mechanisms?" Annual Review of Plant Physiology and Plant Molecular Biology, vol. 51, pp. 167-194, 2000.

[51] O. Mittelsten Scheid, K. Afsar, and J. Paszkowski, "Formation of stable epialleles and their paramutation-like interaction in tetraploid Arabidopsis thaliana," Nature Genetics, vol. 34, no. 4, pp. 450-454, 2003. 

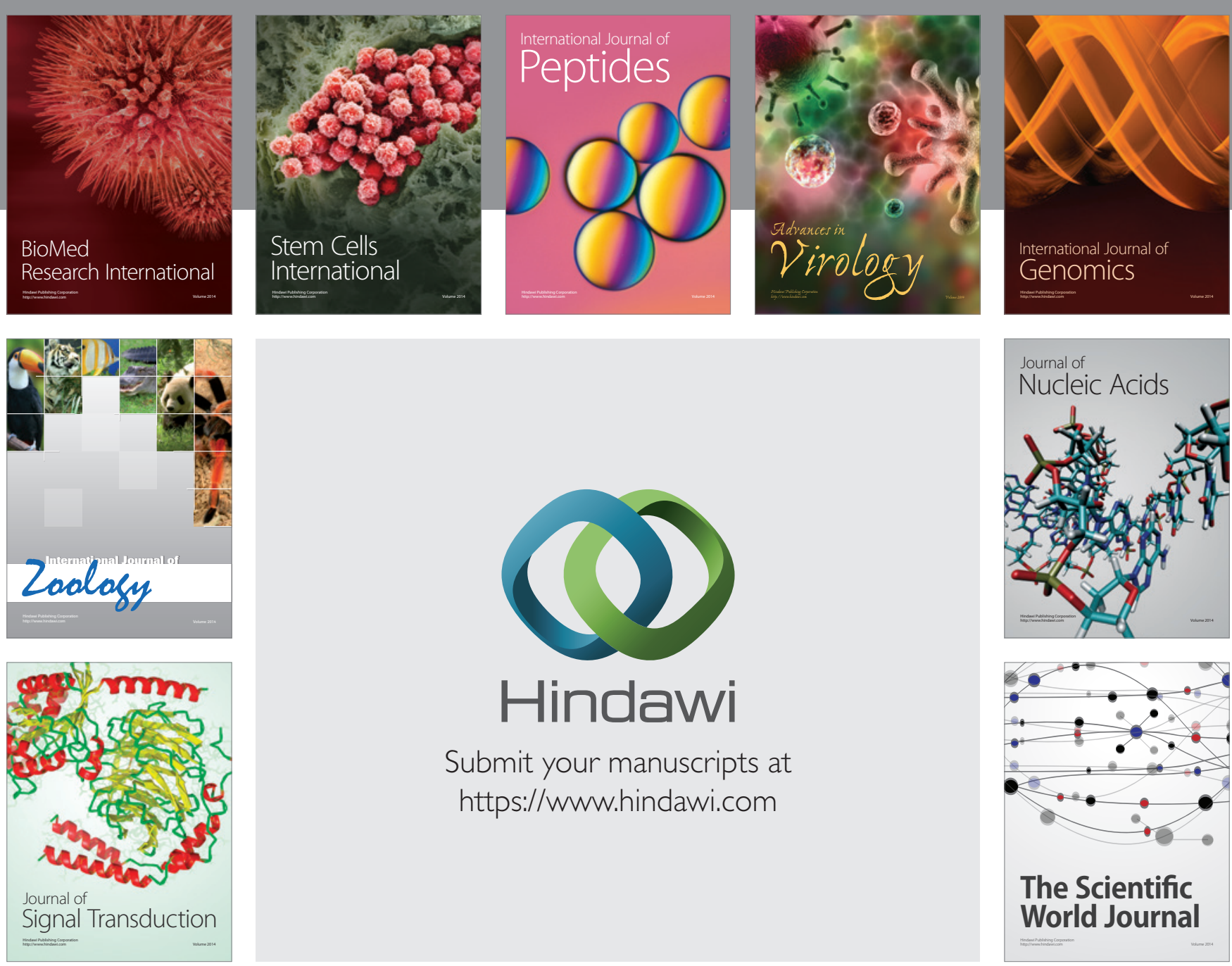

Submit your manuscripts at

https://www.hindawi.com
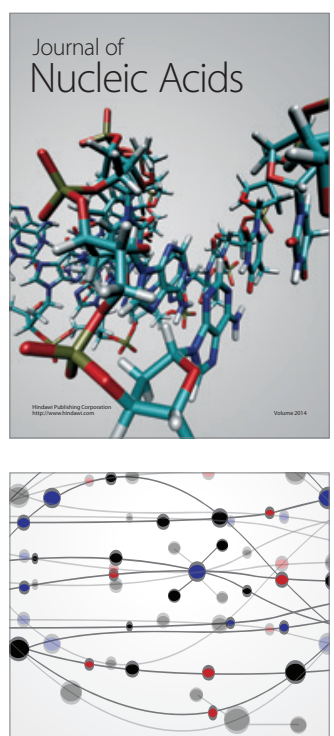

The Scientific World Journal
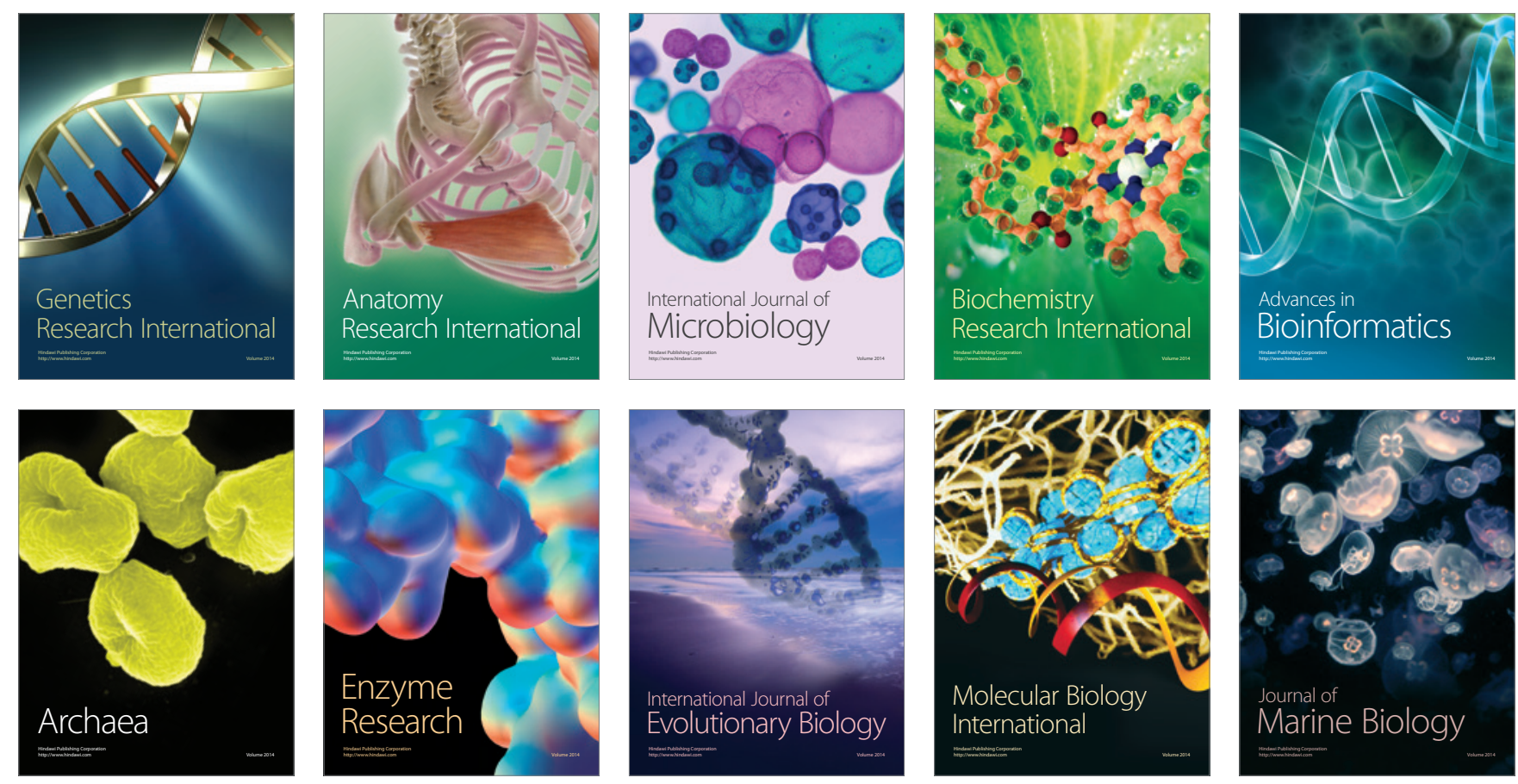